\title{
SOLID STATE ELECTROCHEMICAL ALKALI SOURCES FOR COLD ATOM SENSING
}

\author{
Jonathan J. Bernstein*1, Alison Whale ${ }^{2}$, Justin Brown ${ }^{1,4}$, Cort Johnson ${ }^{l}$, Eugene Cook ${ }^{l}$, Laurent Calvez ${ }^{3}$, \\ Xianghua Zhang $^{3}$ and Steve W. Martin ${ }^{2}$ \\ ${ }^{1}$ Draper, Massachusetts, USA \\ ${ }^{2}$ Iowa State University, USA \\ ${ }^{3}$ University of Rennes, France \\ ${ }^{4}$ Currently at PSI Corp, Massachusetts, USA
}

\begin{abstract}
Alkali sources are used as getters, to coat thermionic emission electrodes and to provide alkali atoms for precision quantum sensors such as clocks, magnetometers, accelerometers and gyroscopes. Commercial alkali sources are electrically heated to $400-700^{\circ} \mathrm{C}$, driving a chemical reaction which produces the alkali. These sources use high current, high power, operate at high temperatures, turn on and off slowly, and evolve impurities. We report here on solid state electrolytic sources for the production of cesium and rubidium at temperatures of $80^{\circ} \mathrm{C}$ to $170^{\circ} \mathrm{C}$. The advantages of electrolytic sources over thermally driven sources are reduced current and power consumption, faster turn-on and turn-off, the ability to monitor the quantity of generated alkali by current integration, and reduced temperature of operation. Resistivity and activation energy of the solid ionic conductors was measured vs. temperature. Alkali vapor pressure was detected by optical absorption in a custom vacuum system. Strong alkali signals (comparable to commercial sources) were measured when current was passed through the samples. Reversing the polarity of the applied voltage results in ambient alkali absorption. These Solid State Ambipolar Alkali Sources (SSAAS) show potential as high purity, precision alkali sources for cold atom sensors where size, weight and power must be reduced.
\end{abstract}

\section{INTRODUCTION}

Alkali sources have been used for decades to coat thermionic emission electrodes and as vacuum getters. Recently, laserinterrogated atomic sensors such as highly accurate clocks [1], magnetometers [2] and inertial sensors [3] have been developed. The most accurate of these sensors operate in a high vacuum, using alkali atoms trapped by magneto-optical traps (MOT's) prior to performing an optical or microwave based measurement of time, magnetic field or acceleration. For these sensors, clouds of $\sim 10^{7}$ atoms are needed. The alkali partial-pressure must be maintained at an acceptable level $\left(\sim 10^{-8}\right.$ to $<10^{-9}$ Torr $)$ over a potentially wide environmental temperature range. The vapor pressure of the pure metals are too high for a solid $\mathrm{Cs}$ or $\mathrm{Rb}$ source to be used. Maintaining the correct background pressure of alkali is further complicated by the kinetics of absorption, adsorption and desorption from the vacuum chamber walls, particularly with glass which can absorb substantial quantities of alkali [4]. An ideal source would be low-power, fast, well-controlled and could also act as a sink to regulate alkali vapor pressure.

Commercial alkali sources from SAES [5] and Alvatec [6] use thermally driven chemical reactions to liberate alkali, and may produce contaminant gasses as well. Typically 4-8 amps is used to resistively heat the device to its operating temperature of $400-700^{\circ} \mathrm{C}$ [7], producing unwanted light, heat and magnetic fields which can interfere with sensor operation.

It has long been known that alkali can be produced by solid state electrolysis of glass [8,9]. Gong et al. [10] demonstrated the

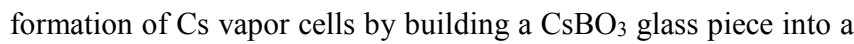
vapor cell wall, and driving $\mathrm{Cs}$ into the cell using a molten salt anode at $325^{\circ} \mathrm{C}$. Later, $\mathrm{Cs}$ generation was demonstrated with $\mathrm{CsBO}_{3}$ glass as an independent source (not part of a vapor cell wall) and using a solid metal anode at $170^{\circ} \mathrm{C}[11]$.

Chalcogenide glasses based on S, Se or Te doped with alkalihalides have been extensively developed for infra-red optics, and are known to have higher ionic mobility than most oxide glasses [12]. Chalcogenide glass is also less hygroscopic than oxide glasses with high alkali content. Na- $\beta$ "'-alumina is a ceramic phase of $\mathrm{Al}_{2} \mathrm{O}_{3}$ and Na with high ionic mobility, which can be substituted for other alkali through a high temperature diffusion process [13].

We report here for the first time chalcogenide (sulfide and selenide) glasses doped with $\mathrm{Cs}$ and $\mathrm{Rb}$ and $\mathrm{Rb}-\beta$-alumina which produce high purity alkali by electrolysis when small currents $(\mu \mathrm{A}$ $\mathrm{mA}$ ) are passed through them.

Figure 1 shows a cartoon of an electrochemical source, with $\mathrm{Rb}$ ions drifting from anode to cathode under a bias voltage. We have also demonstrated for the first time absorption of alkali by reversing the bias voltage of a $\mathrm{Rb} \beta$-alumina source, allowing both emission and absorption of alkali with the same device. A silver anode provides charge compensating ions for the migrating $\mathrm{Rb}$ ions.

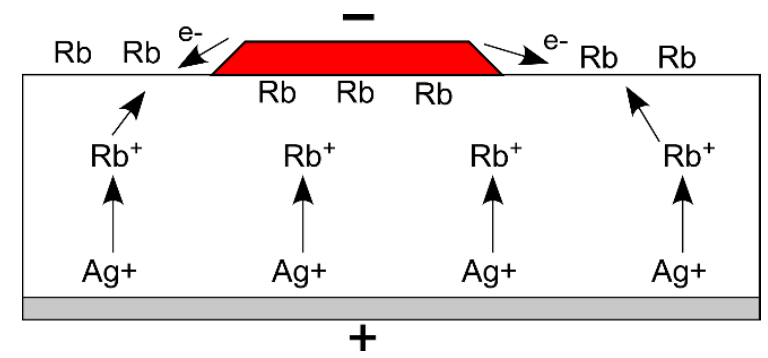

Figure 1. Cross-sectional diagram showing Rb ion migration through source from anode to cathode.

\section{GLASS FABRICATION}

Selenide glass $\left(\mathrm{GeSe}_{2}+\mathrm{Ga}_{2} \mathrm{Se}_{3}\right)$ samples containing $30 \%$ or $40 \% \mathrm{CsI}$, and sulfide glass containing $\mathrm{CsCl}$ were produced at Iowa State University (ISU). Selenide glass with $25 \%$ or $30 \%$ RbI was produced at the University of Rennes. Rb-substituted $\beta$-alumina was obtained from Ionotec.

The glass samples from ISU were prepared from appropriate amounts of $99.99 \%+$ purity constituent elements ( $\mathrm{Ge}, \mathrm{Ga}, \mathrm{Se}, \mathrm{CsCl}$ or CsI) then sealed in evacuated silica ampules. The silica glass ampules were placed in a rocking furnace and heated at $1{ }^{\circ} \mathrm{C} / \mathrm{min}$ to $850-950^{\circ} \mathrm{C}$ and then held for 12 hours. Different soaking temperatures were used to determine the optimum temperature. It was found that $950^{\circ} \mathrm{C}$ provided a high enough temperature to ensure complete reaction among the elements and yet not so high that vapor pressure would break the sealed glass tube. After soaking for 12 hours, the ampule was immediately transferred to a furnace that was 
preheated to the glass annealing temperature of 250 to $270^{\circ} \mathrm{C}$ for four hours.

\section{ELECTRODING}

Anode: The back anode needs to inject mobile ions into the glass to avoid building up space charge as the alkali ions migrate up towards the cathode. This has proved a problem in previous work. In [10] a molten $\mathrm{NaNO}_{3}$ salt anode was used as a source of compensating ions. Developers of high temperature alkali ion guns [14] using ion-exchanged Cs-mordenite electrolyte found that current flow was limited by anodic processes causing oxidation of the host lattice. Space charge formation led to a rise in voltage necessary to drive current through their device.

It is well known that metals such as $\mathrm{Ag}$ and $\mathrm{Cu}$ form nonblocking electrodes which inject ions into glass and have high ionic mobility [15]. For this reason continuous Ag electrodes were chosen for our anodes. The use of solid ion-injecting electrodes prevents development of space charge regions or oxidation of the host lattice anions.

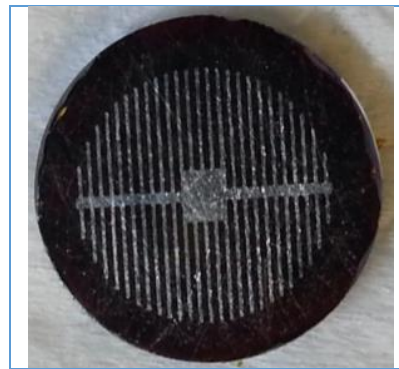

Figure 2. CsI-GeSe $2-\mathrm{Ga}_{2} \mathrm{Se}_{3}$ glass source with Al front electrodes.

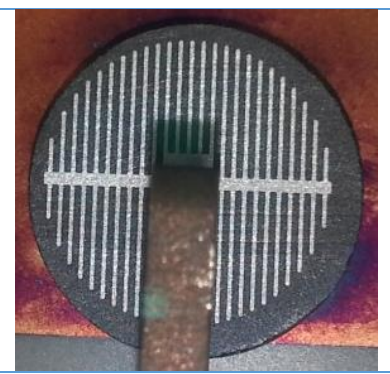

Figure 3. RbI-GeSe $2-\mathrm{Ga}_{2} \mathrm{Se}_{3}$ glass source and spring contact.
Cathode: Cathodes were applied by evaporation of $\mathrm{Al}$ through an etched Invar shadow mask, held in place by magnets. The role of the cathode is to supply electrons to neutralize the alkali ions migrating to the surface. To allow the alkali atoms to evaporate without being blocked by the metal fingers, it is desirable to make these fingers narrow and to cover a small fraction of the front surface. Figures 2, 3 and 5 shows samples after application of the cathode finger array. These samples are 10-14 mm diameter and 1$3 \mathrm{~mm}$ thick. The shadow mask has finger widths resulting in 160 $\mu \mathrm{m}$ wide metal lines with 400 um spacing, or about $60 \%$ open area.

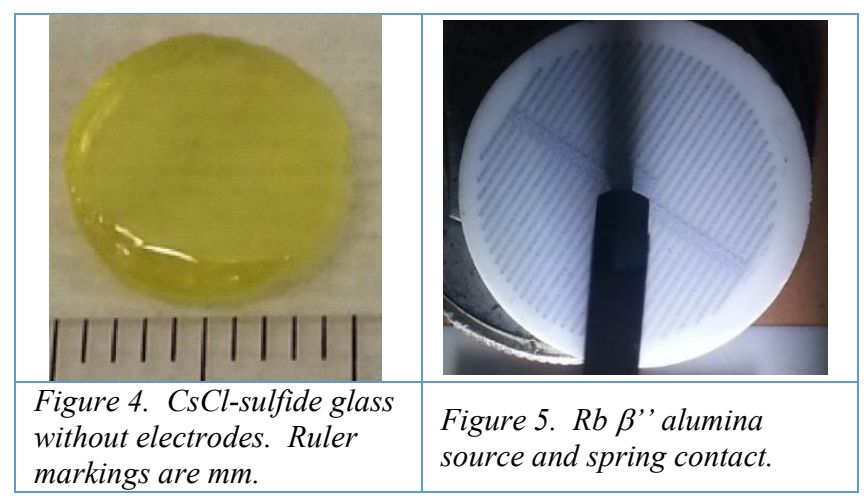

\section{EXPERIMENTAL METHODS}

A vacuum chamber was constructed from a 6 port stainless steel cube for the measurement of alkali generation. A mechanically backed turbo-pump was used for evacuation to the low $10^{-7}$ Torr range after bake-out. This pump could be valved off and an ion pump used to maintain vacuum.

A multi-pin electrical feedthrough to the chamber has connections for a small ceramic heater with built-in thermocouple, high voltage and ground to drive the solid state source, and driving a commercial source from SAES or Alvatec, used for demonstrating alkali absorption.

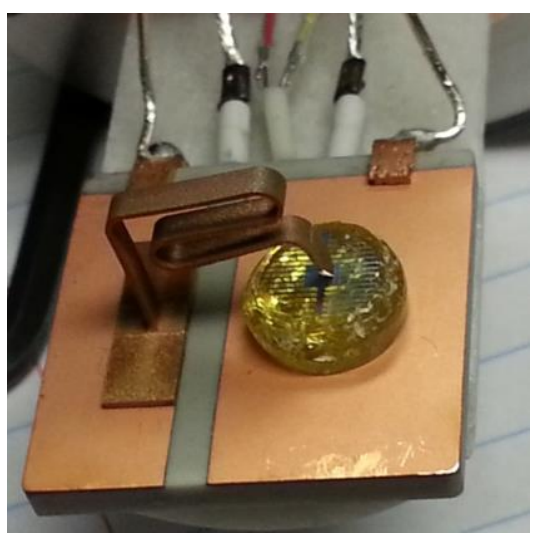

Figure 6. $25 \mathrm{~mm} X 25 \mathrm{~mm}$ heater, CsCl-sulfide glass sample and Be-Cu spring clip.

Alkali Measurement: A diode laser tuned to an optical transition of the alkali species of interest is passed through the chamber just above the sample to measure the concentration of each alkali $[\mathrm{Cs}]$ or $[\mathrm{Rb}]$. To detect small absorption signals we used a lock-in detection method, the block diagram of which is shown in Figure 7. Phase modulation is applied using an electro-optic modulator (EOM) to put $2 \mathrm{GHz}$ sidebands on laser light locked to an atomic transition. The EOM is driven almost to carrier suppression such that most of the optical power is in the sidebands. These sidebands are sufficiently detuned from the Doppler broadened atomic transition $(\sim 1 \mathrm{GHz})$ to pass through the vapor with minimal absorption. An RF switch driven with a $32 \mathrm{kHz}$ square wave gates between light on-resonance and (modulated) light off-resonance.

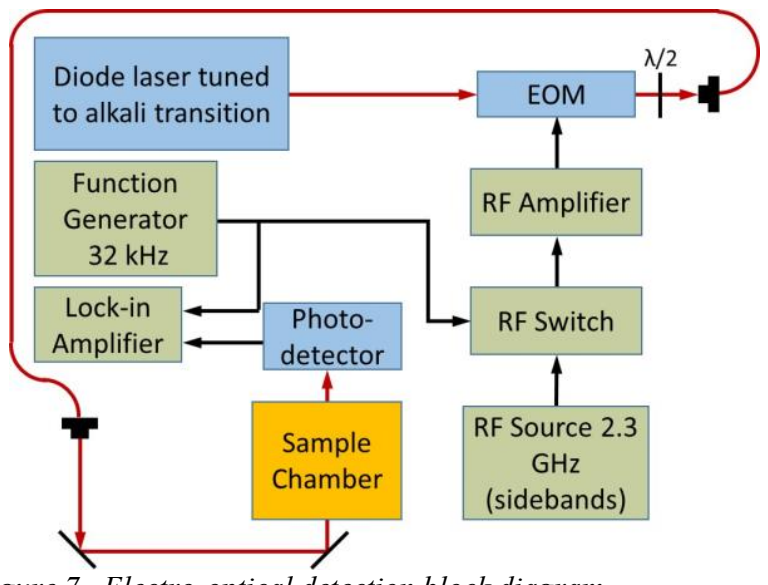

Figure 7. Electro-optical detection block diagram.

A lock-in amplifier referenced to the $10 \mathrm{kHz}$ modulation provides the change in amplitude due to the absorption. The sensitivity of the measurement is currently limited by residual amplitude modulation of the EOM to Cs vapor densities of 
$\sim 1 \times 10^{8} / \mathrm{cm}^{3}$. The detected alkali density is calibrated using standard alkali vapor cells (e.g. Thorlabs GC25075-CS).

\section{CONDUCTIVITY MEASUREMENTS}

The alkali ion conductivity vs. temperature was measured at ISU by small signal AC impedance techniques extrapolated to DC, after applying and baking silver paint electrodes on both sides. Results for 6 samples (5 material compositions) are shown in Figure 8. The $\beta$ " alumina has the highest conductivity. The $\mathrm{Rb}$ and $\mathrm{Cs}$ doped selenide glasses have a conductivity which depends strongly on the concentration of alkali [12], although activation energy for the various glass samples is similar at $\sim 0.77 \mathrm{eV}$. High conductivity is an advantage as it allows operation at lower voltages and temperatures.

DC current-voltage vs. temperature measurements were made using evaporated $\mathrm{Al}$ finger electrodes on the front, and continuous silver electrodes on the back. Due to various effects including current crowding, space charge and electrode interfacial reactions, the ionic conductivity measured this way is typically $100 \mathrm{X}$ lower than the small signal AC measurements. A plot of current-voltage vs. temperature for a $\mathrm{CsCl}$-sulfide glass is shown in Figure 9.

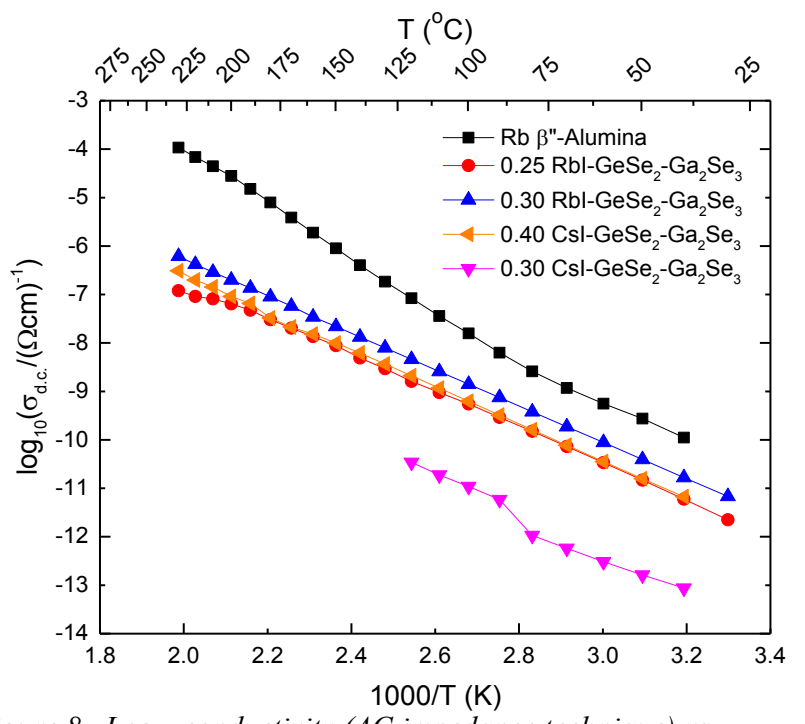

Figure 8. $\log _{10}$ conductivity (AC impedance technique) vs. 1000/T(K) for 5 materials.

\section{ALKALI EMISSION}

Samples were placed on the ceramic heater in the vacuum system, held in place by a $\mathrm{Be}-\mathrm{Cu}$ spring which also connects electrically to the top finger array. The resonant laser was directed over the top surface of the glass sample and its absorption was measured to determine the alkali vapor concentration. Alkali density was calibrated by comparison with the absorption of standard $\mathrm{Cs}$ or $\mathrm{Rb}$ vapor cells maintained at a known temperature.

CsCl-Sulfide Glass: A sample of $\mathrm{CsCl}-\mathrm{GeSe}_{2}-\mathrm{Ga}_{2} \mathrm{Se}_{3}$ glass from ISU was metallized with $\mathrm{Ag}$ on the back and $\mathrm{Al}$ fingers on the front. Current-voltage curves with temperature as a parameter were measured, shown in Figure 9. The current roughly doubles for every $10^{\circ} \mathrm{C}$ increase in temperature.

Cs vapor emission from this sulfide glass as measured by laser absorption is shown in Figure 10 , held at $130^{\circ} \mathrm{C}$ with a bias voltage of $1200 \mathrm{~V}$ and current of $5 \mu \mathrm{A}$, cycled on and off twice. Cs losses are primarily to the chamber walls and (much less) to the pumps.

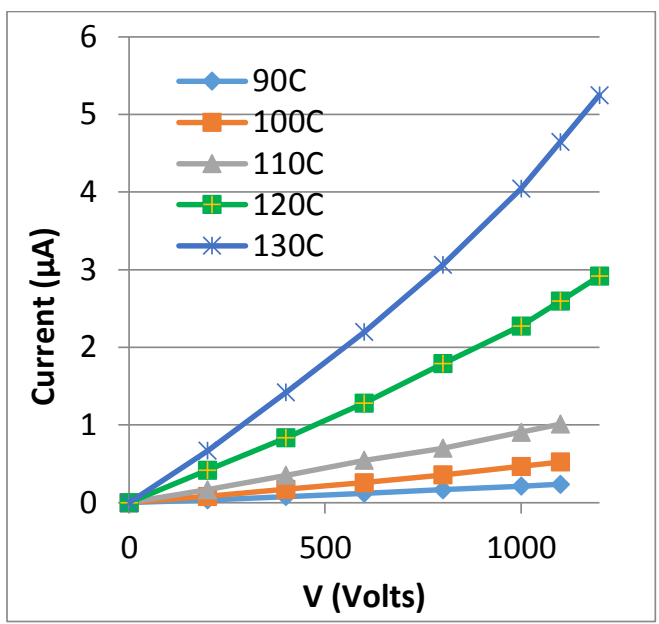

Figure 9. DC current-voltage-temperature plot for CsCl-Ge-Gasulfide glass shown in Figure 4.

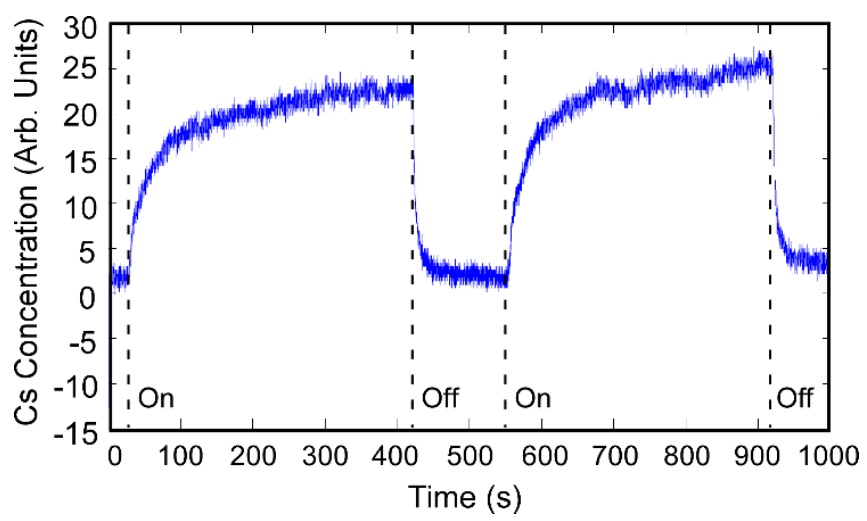

Figure 10. Optical absorption signal for Cs emission from sulfide glass sample at $130^{\circ} \mathrm{C}$. Current through sample is $5 \mu \mathrm{A}$.

CsI-GeSe $-\mathrm{Ga}_{2} \mathrm{Se}_{3}$ Glass: A 40\% CsI doped selenide glass supplied by ISU was electroded with Al fingers (cathode) and continuous $\mathrm{Ag}$ or $\mathrm{Cu}$ (anode). Strong $\mathrm{Cs}$ generation signals were measured after biasing to $1 \mathrm{kV}$ at $170^{\circ} \mathrm{C}$ and $44-50 \mu \mathrm{A}$ (Figure 11).

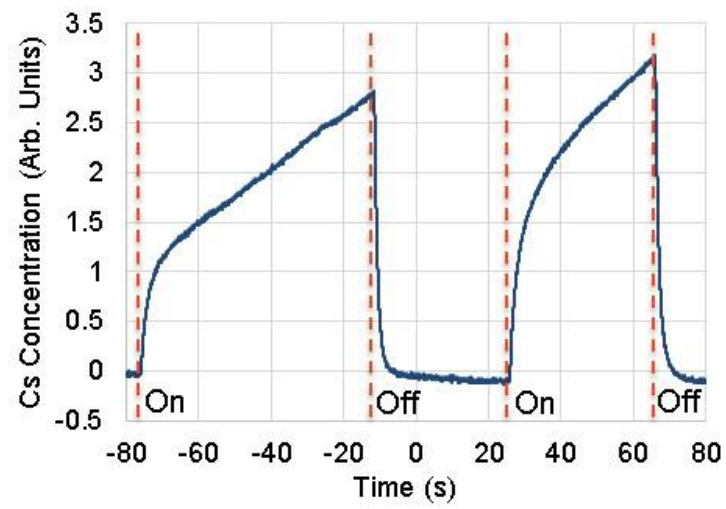

Figure 11. Cs generation from $\mathrm{CsI}-\mathrm{GeSe}_{2}-\mathrm{Ga}_{2} \mathrm{Se}_{3}$ glass.

Kinetics of alkali absorption and desorption from the chamber walls largely determine the concentrations and rate of change of the measured alkali in vacuum. Curve fitting the decay time of the $\mathrm{Cs}$ concentration shown in (e.g. Figure 11) gave a value of $1.25 \mathrm{~s}$ regardless of whether the chamber is sealed or actively pumped, 
because at low pressure the chamber walls dominate the alkali partial pressure kinetics. Due to chamber wall effects, we do not yet know how fast the solid state sources can be turned on and off, but it is likely much faster than the present generation of thermally driven sources [16].

RbI GeSe2-Ga2Se 3 Glass: A RbI doped glass supplied by the University of Rennes was electroded in a similar manner to the previous samples (Al cathode fingers and continuous $\mathrm{Ag}$ back anode). Strong $\mathrm{Rb}$ absorption signals were seen when the glass was forward biased at $170^{\circ} \mathrm{C}$ and $1 \mathrm{kV}$, (Figure 12). Current through the sample increased from $80 \mu \mathrm{A}$ to $250 \mu \mathrm{A}$ at constant voltage, which is most likely due to sample self-heating. A constant current drive would provide improved stability compared to constant voltage.

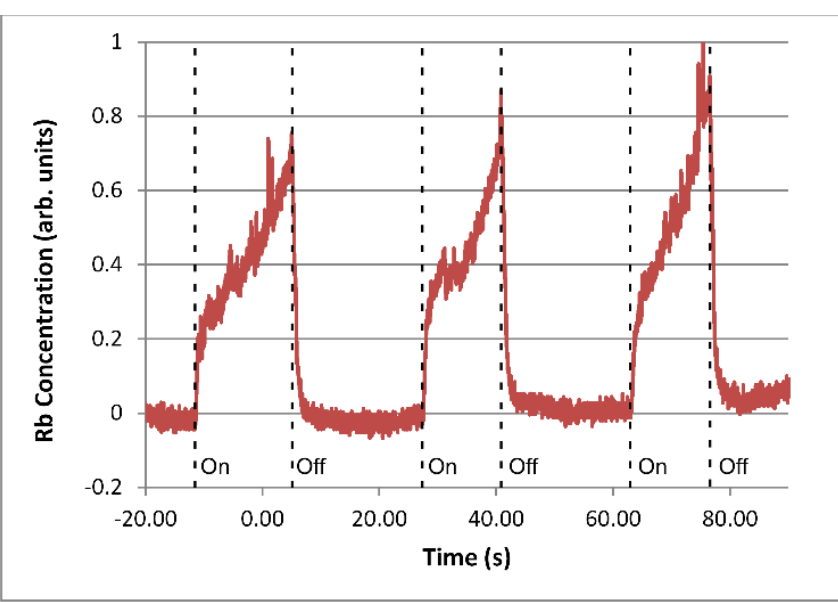

Figure 12. Rb generation signal from $30 \%$ RbI doped selenide glass.

$\boldsymbol{\beta}$ " Alumina Ceramic: Figure 13 shows Rb emission from a $\mathrm{Rb} \beta$ " alumina sample biased at 50 volts and a current of $0.7 \mathrm{~mA}$ at $120^{\circ} \mathrm{C}$. Calibration of the atom density was performed by comparison with a standard $\mathrm{Rb}$ filled ampule. The strength of the $\mathrm{Rb}$ signal from this source was comparable to that from a SAES Rb source driven at 5 amps. The higher mobility of $\mathrm{Rb}$ in the $\beta$," alumina allows this source to operate at reduced temperature and voltage.

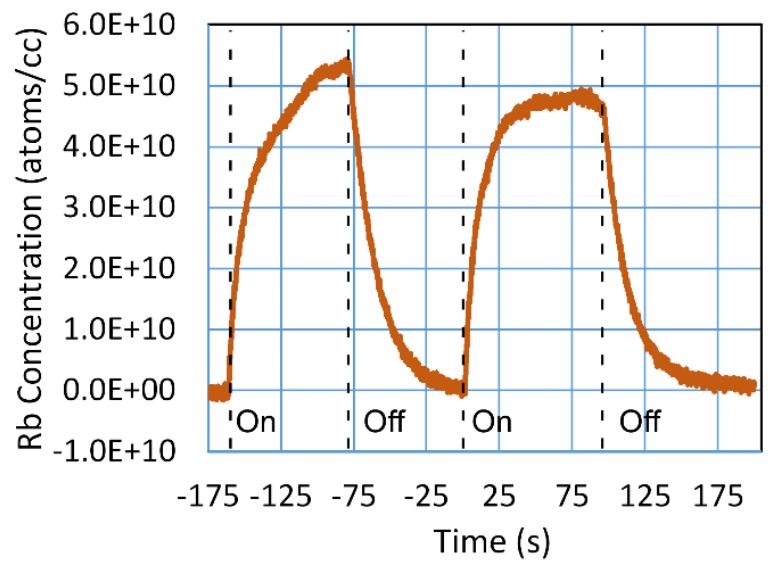

Figure 13. Rb generation from Rb doped $\beta$ ' alumina.

Alkali Absorption: By reverse biasing the device, a surface region depleted of alkali ions is created. This ion-depletion region is capable of absorbing incident alkali atoms, ionizing them and driving ions into the bulk. To demonstrate absorption, a Rb SAES source was mounted in the chamber and run at 4.88 amps for several minutes to establish a constant background $\mathrm{Rb}$ pressure. $\mathrm{A} \mathrm{Rb}$ doped $\beta$ " alumina source was then reverse biased at $-130 \mathrm{~V}(-0.21$ $\mathrm{mA}$ ) and $130^{\circ} \mathrm{C}$, creating a $\mathrm{Rb}$ depleted surface while the SAES source was left on. The measured $\mathrm{Rb}$ concentration was reduced by $30-50 \%$, as shown in Figure 14. Considering the small size of the SSAAS device $\left(\sim 1.1 \mathrm{~cm}^{2}\right)$ compared to the chamber walls $(>280$ $\mathrm{cm}^{2}$ ), this demonstrates remarkably efficient absorption.

The measured $\mathrm{Rb}$ concentration is an integral over the path of the laser through the vacuum system. At each point in the path, the vapor pressure is proportional to an average over the entire $4 \pi$ steradians solid angle of the incident flux of alkali atoms emitted from the surrounding surfaces. The laser passes close to the surface of the SSAAS device, and for that part of the integral, the SSAAS device is almost half the solid angle.

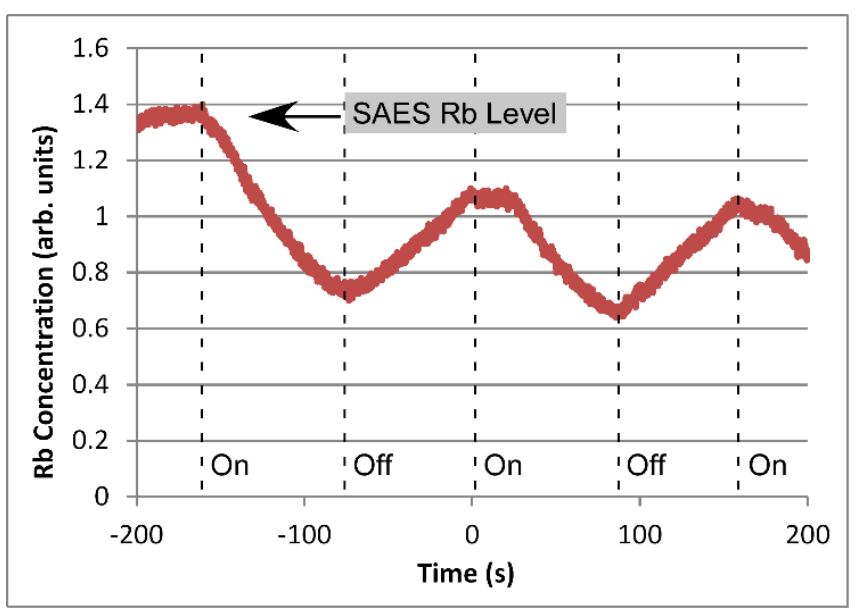

Figure 14. SSAAS device reverse biased as absorber. Background level set by SAES source at 4.88 A. SSAAS absorber turned on and off several times is able to absorb $50 \%$ of the $R b$ produced by the SAES source.

\section{CONCLUSIONS}

We have demonstrated solid state electrochemical alkali sources which, when compared to commercial thermally driven chemical sources, require greatly reduced current and power, operate at lower temperatures $\left(70-170^{\circ} \mathrm{C}\right.$, compared to $\left.400-700^{\circ} \mathrm{C}\right)$, exhibit faster turn-on and turn-off, and can both source and sink alkali using direct current control. The output of alkali was demonstrated to be comparable in magnitude to commercial SAES alkali sources consuming 5 amps.

Cold atom clouds formed in Magneto-Optical Traps (MOT's) used in high precision atomic clocks, magnetometers and inertial sensors require only about $10^{6}$ to $10^{8}$ atoms in a typical measurement. Solid state electrochemical sources will reduce the power, current and temperature required to produce alkali atoms by orders of magnitude. As cold-atom sensors enter the marketplace they will benefit from the high purity, small size and low power offered by these novel solid state atom sources.

\section{ACKNOWLEDGEMENTS}

The authors gratefully acknowledge funding from the DARPA Cold Atom MicroSystems (CAMS) Solid State Ambipolar Alkali Source 
(SSAAS) program (contract \# HR0011-14-C-0115). We also acknowledge Christopher Roper for bringing Rb- $\beta$ " alumina to our attention. Mitchel Hansberry is thanked for mechanical design and Joseph Louis for metallization engineering. The views expressed

\section{REFERENCES}

1. D. Meekhof, S. Jefferts, M. Stepanovic and T. Parker, "Accuracy Evaluation of a Cesium Fountain Primary Frequency Standard at NIST", IEEE Trans. Instrumentation and Measurement, Vol. 50, NO. 2, April 2001.

2. D. Budker and M. Romalis, "Optical Magnetometry", Nature Physics 3, 227 - 234 (2007).

3. M. Gilowski, C. Schubert, T. Wendrich P. Berg, G. Tackman, W. Ertmer and E. Rasel, "High resolution rotation sensor based on cold Rubidium atoms", IEEE International Frequency Control Symposium 2009, pp. 1173-1175, ISBN 978-1-4244-3511-1.

4. J. Ma, A. Kishinevski, Y.-Y. Jau, C. Reuter, and W. Happer, "Modification of glass cell walls by rubidium vapor", Phys. Rev. A 79, 042905 (2009).

5. https://www.saesgetters.com/products/alkali-metals-dispensers

6. http://www.alvatec.com/products/

7. M. Succi, R. Canino and B. Ferrario, "Atomic absorption evaporation flow rate measurements of alkali metal dispensers", Vacuum, Vol. 35 Number 12 pp. 579-582 (1985).

8. E. Warburg and F. Tegelmeyer, Ann. Phys. (Weidemann Series) 35,455 (1888).

9. D.F. Klemperer and I. Mackenzie, "Vacuum preparation of pure cesium by electrolysis of glass", Journ. of Vac. Sci. and Techn., Vol. 13, No. 6, pp. 1166-1171 (1976). are those of the authors and do not reflect the official policy or position of the Department of Defense or the U.S. Government.

10. F. Gong, Y.-Y. Jau, K. Jensen, and W. Happer, "Electrolytic fabrication of atomic clock cells", Rev. Sci. Instruments 77, 076101 (2006).

11. J. Bernstein, S. Feller, A. Ramm, J. North, J. Maldonis, M. Mescher, W. Robbins, R. Stoner, and B. Timmons, "All solid state ion-conducting cesium source for atomic clocks", Journal of Solid State Ionics, Volume 198, Issue 1, 19 Sept. 2011, Pages 47-49.

12. A. Pradel and M. Ribes, "Ionic conductivity of chalcogenide glasses", pp. 169, Chapter 7 in "Chalcogenide glasses, preparation, properties and applications", Ed. J.L. Adam and X. Zhang, Woodhead Publishing, Cambridge, 2014.

13. R.H. Radzilowski, Y.F. Yao, and J.T. Kummer, "Dielectric Loss of Beta Alumina and of Ion-Exchanged Beta Alumina", Journal of Applied Physics, Volume 40, Issue 12, p.4716-4725.

14. S.I. Kim and M. Seidl, "A New Solid-State Cesium Ion Source", J. Appl. Phys. 67 (6), 15 March 1990, pp. 2704-2710.

15. E. Bychkov, "Superionic and ion-conducting chalcogenide glasses: Transport regimes and structural features", Solid State Ionics 180 (2009) pp. 510-516.

16. J. Fortagh, A. Grossmann, T. W. Hänsch, and C. Zimmermann, "Fast loading of a magneto-optical trap from a pulsed thermal source", Journal of Applied Physics 84, 6499 (1998); doi: $10.1063 / 1.369018$

\section{CONTACT}

J. J. Bernstein, tel: +1 617-258-2513, jbernstein@draper.com 